\title{
Extended Half-Life Factor VIII/Factor IX Products: Assay Discrepancies and Implications for Hemophilia Management
}

\author{
Jens Müller ${ }^{1 *}$ Georg Goldmann ${ }^{1} \quad$ Natascha Marquardt $^{1} \quad$ Bernd Pötzsch $^{1} \quad$ Johannes Oldenburg ${ }^{1}$ \\ ${ }^{1}$ University Hospital Bonn, Institute of Experimental Haematology \\ and Transfusion Medicine, Bonn, Germany \\ Hämostaseologie 2020;40(Suppl 1):S15-S20. \\ Address for correspondence Jens Müller, PhD, University Hospital \\ Bonn, Institute of Experimental Haematology and Transfusion \\ Medicine, Venusberg-Campus 1, 53127 Bonn, Germany \\ (e-mail: jens.mueller@ukbonn.de).
}

\section{Abstract \\ Keywords \\ - hemophilia A/B \\ - factor concentrates \\ - extended half-life products \\ - prophylaxis}

Zusammenfassung

\section{Schlüsselwörter}

- Hämophilie A/B

- Faktorenkonzentrate

- Verlängerte Halbwertszeit

- Prophylaxe
Due to structural differences between extended half-life (EHL) factor VIII (FVIII) or FIX products and equivalent plasma wild-type molecules used for assay calibration, reagent-dependent discrepancies during monitoring of FVIII- and FIX-replacement therapies with EHL products have been described. To assess the performance of available one-stage clotting and chromogenic substrate assays on the Siemens Atellica COAG 360 analyzer, an in vitro study using spiked plasma samples was performed. The described results confirm previously described findings and allowed allocation of each EHL product to an appropriate assay. In addition, corresponding EHL product-specific analytes were defined within the order entry system of the University Hospital Bonn. The requirement of product-specific FVIII and FIX assays complicates patient monitoring and demonstrates the need for both continuous education and communication between treating physicians and the coagulation laboratory.

Aufgrund struktureller Unterschiede zwischen FVIII- und FIX-Produkten mit verlängerter Halbwertszeit (EHL) und den zur Kalibration der Testsysteme verwendeten plasmatischen Faktoren kommt es zu reagenzabhängigen Diskrepanzen von im Rahmen der Therapie mit EHL-Konzentraten erhobenen Aktivitätswerten. Zur entsprechenden Überprüfung der uns auf dem Atellica Coag 360 Analysensystem zur Verfügung stehenden Einstufen- und chromogenen Testverfahren wurde eine in-vitro-Studie mit entsprechend präparierten Plasmaproben durchgeführt. Die gefundenen und hier beschriebenen Ergebnisse bestätigen zuvor publizierte Daten und erlaubten die Zuordnung der einzelnen EHL-Produkte zu einem jeweils geeigneten Testverfahren. Die produktspezifischen Testverfahren wurde durch die Anlage entsprechender Analyte im Order-Entry-System des Universitätsklinikums Bonn (UKB) hinterlegt. Die Notwendigkeit produktspezifischer FVIII- und FIX-Tests kompliziert das Patientenmonitoring und verdeutlicht die Notwendigkeit entsprechender Fortbildungen als auch einer guten Kommunikation zwischen behandelndem Arzt und Labor. received

September 15, 2020

accepted

October 5, 2020 (c) 2020 Georg Thieme Verlag KG Stuttgart · New York
DOI https://doi.org/

$10.1055 / \mathrm{a}-1282-2251$. ISSN 0720-9355. 


\section{Introduction}

Hemophilia A and B are X-linked hereditary bleeding disorders caused by lack of activity of coagulation factors VIII (FVIII) and IX (FIX), respectively. ${ }^{1}$ Historically, the treatment of hemophilia evolved from the transfusion of whole blood, fresh frozen plasma, and cryoprecipitate to targeted replacement therapies with plasma-derived FVIII or FIX concentrates. $^{2,3}$ Alongside this development, two distinct principles for the measurement of plasma FVIII or FIX activities have been established in coagulation laboratories worldwide, the activated partial thromboplastin time (aPTT)-based one-stage clotting (OSC) and chromogenic substrate (CS) assays, both, to date, usually calibrated against plasma FVIII or FIX standards. ${ }^{4}$ It has been known for a long time that about $30 \%$ of patients with nonsevere hemophilia A show mutation-specific differences between these assays. $^{5-7}$ With the advent of recombinant factor concentrates, the molecular structures of the substituted factors started to differ from that present in the plasma calibrators, leading to discrepant results, mainly, however, limited to differences between the OSC and the CS assay formats. ${ }^{8-10}$ This situation has been further complicated by the introduction of modified recombinant factor concentrates with extended half-life (EHL; typically $\sim 1.5$-fold for FVIII and $\sim 5$-fold for FIX) that additionally cause aPTT reagent-dependent assay discrepancies. ${ }^{11,12}$ In the present in vitro study, we assessed the accuracy of different OSC and CS assays with respect to the analysis of available EHL-FVIII and EHL-FIX concentrates on the Siemens Atellica COAG 360 analyzer. ${ }^{13}$ The results are discussed in light of the recent literature and illustrate the need for EHL-FVIII-/FIX productspecific laboratory requests to adequately address observed assay discrepancies.

\section{Materials and Methods}

\section{Extended Half-Life Factor VIII/Factor IX Concentrates and Assay Reagents}

Damoctocog alfa pegol (Jivi) was provided by Bayer Vital $\mathrm{GmbH}$ (Leverkusen, Germany). Lonoctocog alfa (Afstyla) and albutrepenonacog alfa (Idelvion) were made available by CSL Behring (Marburg, Germany). Turoctocog alfa pegol (Esperoct) and nonacog beta pegol (Refixia) were provided by Novo Nordisk (Bagsværd, Denmark). Efmoroctocog alfa (Elocta) and eftrenonacog alfa (Alprolix) were made available by Sobi (Stockholm, Sweden). Rurioctocog alfa pegol (Adynovi) was provided by Takeda (Konstanz, Germany).

The aPTT reagents Actin FS (AFS), Actin FSL (AFSL), and Pathromtin SL (PSL); the FVIII CS assay (FVIII chromogenic), FVIII-, and FIX-deficient plasma; as well as standard human plasma (SHP; containing defined plasma (p)FVIII and pFIX activities) and controls were available from Siemens Healthcare Diagnostics (Marburg, Germany). The FIX CS assay (BIOPHEN Factor IX) and corresponding plasma calibrator (PCal) and controls were purchased from Hyphen-Biomed (Neuville-sur-Oise, France).

\section{Preparation and Analysis of Spiked Plasma Samples}

EHL concentrates were dissolved according to the manufacturer's instructions and diluted in the respective factor-deficient plasma to achieve the following final concentrations: 80, 25.3, 8, and $2.53 \mathrm{IU} / \mathrm{dL}$ (according to batch-specific contents). Subsequently, the prepared samples were analyzed for FVIII or FIX activities using the Atellica Coag 360 system (Siemens Healthcare Diagnostics). The single assays were performed as specified by the manufacturer and calibrated against SHP (Siemens OSC and CS assays) or PCal (Hyphen-Biomed FIX CS assay).

\section{Presentation and Interpretation of Data}

Measured FVIII or FIX activities were plotted relative to input concentrations using vertical column charts. A general acceptance range of $\pm 20 \%$ (80-120\%) was defined and presented as horizontal lines. Assays/reagents that led to relative results within this arbitrary acceptance range were deemed (best) suitable for the determination of plasma EHL-factor activities.

\section{Results}

\section{Extended Half-Life for Hemophilia A Treatment}

Measured FVIII:C activities relative to EHL product input concentrations are described below and summarized in -Fig. 1.

Efmoroctocog Alfa (Elocta, Extended Half-Life Factor VIII) Efmoroctocog alfa (Elocta) is a recombinant fusion protein of a B-domain-deleted (BDD) FVIII molecule and the Fc fragment of human immunoglobulin G1 (IgG1). ${ }^{14}$ As demonstrated in - Fig. 1A, this modification showed only a minor impact on SHP-calibrated OSC assay accuracy. All one-stage FVIII assays showed a FVIII:C in the expected range. Using the FVIII CS assay, however, a concentration-dependent overestimation of assay results was found.

Damoctocog Alfa Pegol (Jivi, Extended Half-Life Factor VIII) Damoctocog alfa pegol (Jivi) is a recombinant BDD (rBDD) FVIII molecule with site-specific 60-kDa PEG molecule. ${ }^{15}$ AFS and AFSL showed results in the expected range, but AFSL underestimated Jivi at the tested low concentration ( - Fig. 1B). PSL tended to overestimate corresponding FVIII activities. In contrast to the OSC assays, Jivi was found to be significantly underestimated by the applied FVIII CS assay (-Fig. 1B).

\section{Lonoctocog Alfa (Afstyla, Single-Chain Factor VIII)}

Lonoctocog alfa (Afstyla) is a recombinant B-domain-truncated single-chain (sc) FVIII molecule that, in comparison to wildtype (wt) FVIII, shows tighter binding to von Willebrand factor (vWF), leading to increased stability of the molecule in circulation. ${ }^{16}$ These characteristics result in a prolonged half-life of the molecule ( $\sim 1.2$-fold), Afstyla. ${ }^{11}$ As shown in - Fig. 1C, the scFVIII molecule was underestimated by all aPTT reagents in a concentration-dependent manner, while only the FVIII CS assay yielded acceptable results. With respect to the OSC FVIII:C assays, CSL Behring recommends to multiply the measured FVIII:C by 2; however, the preferred strategy should be to use upfront the best fitting FVIII:C assay, which is the FVIII CS assay. 

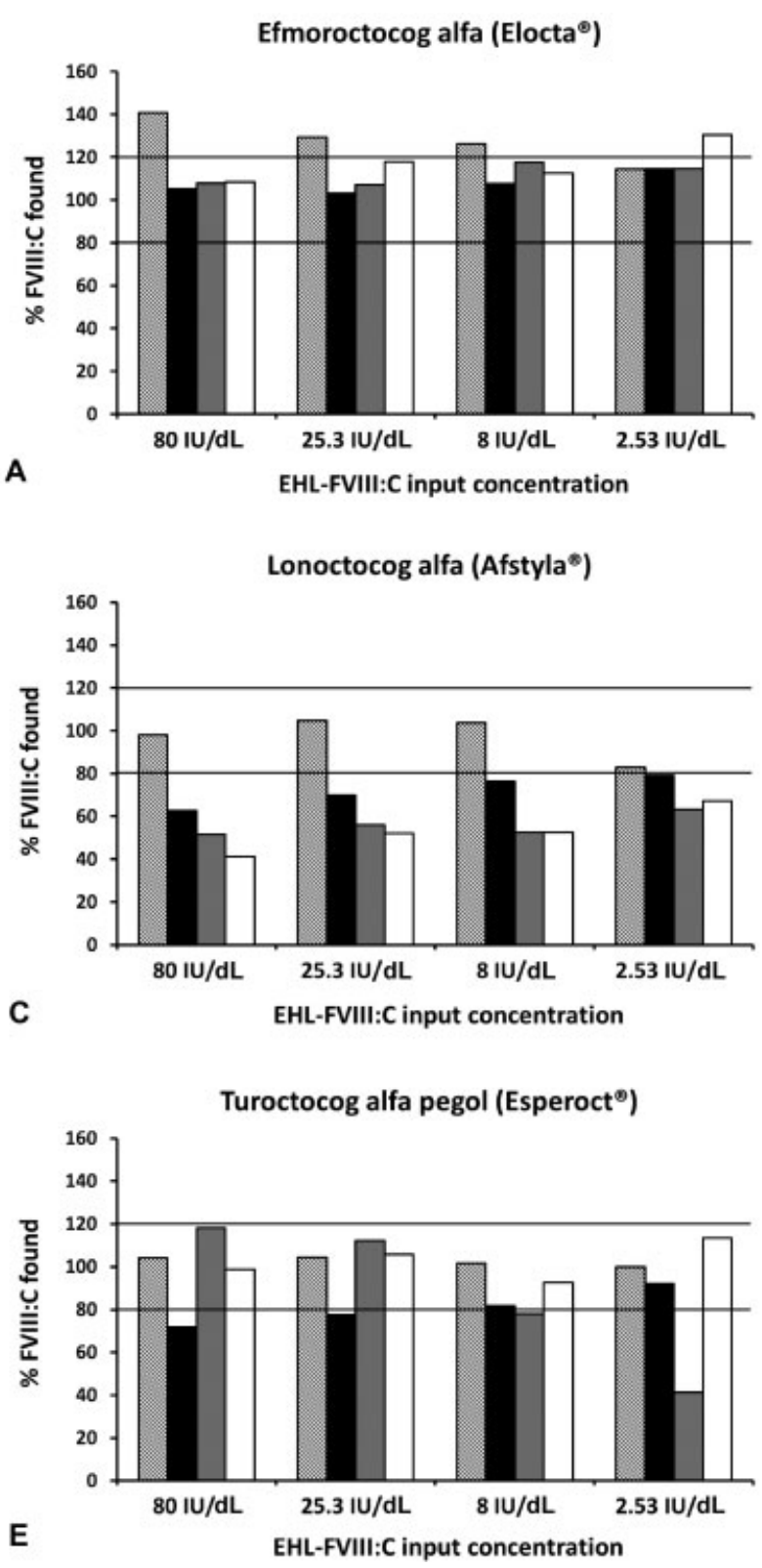
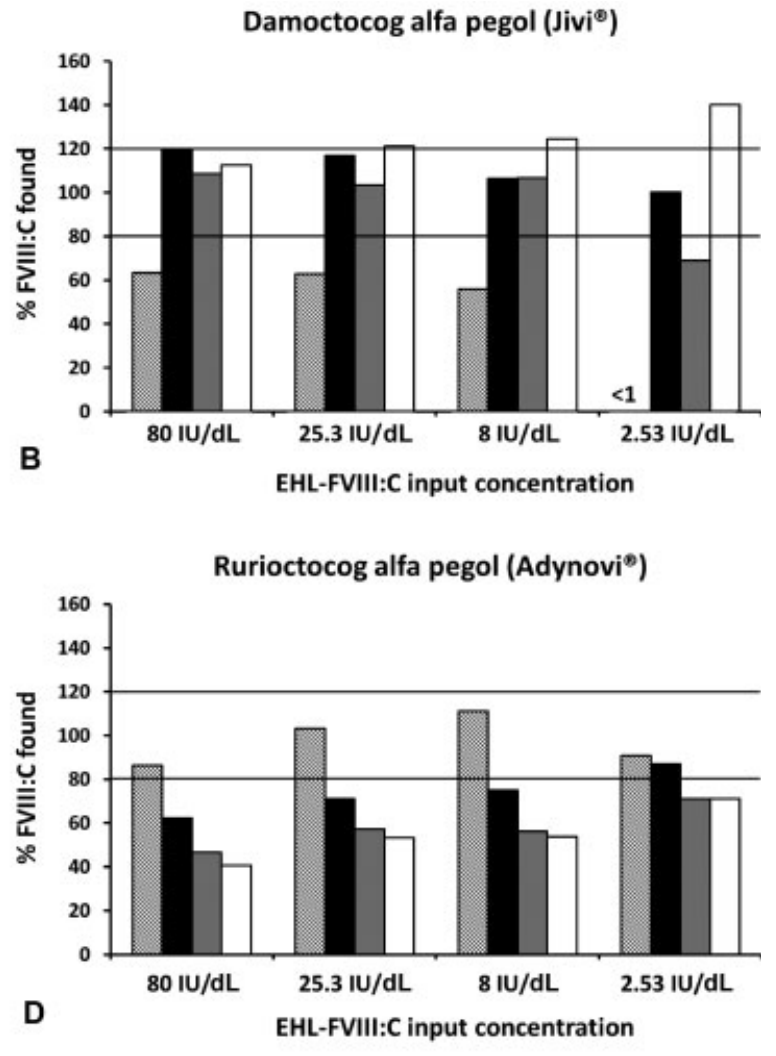

\section{Rurioctocog Alfa Pegol (Adynovi, Extended Half-Life Factor VIII)}

Rurioctocog alfa pegol (Adynovi) is a full-length recombinant FVIII molecule conjugated with a polyethylene glycol (PEG) reagent, predominantly attached to the B-domain of the molecule. ${ }^{17}$ Comparable to the results found for lonoctocog alfa (Afstyla), the molecule was underestimated by all aPTT reagents in a concentration-dependent manner, while the FVIII:C CS assay yielded acceptable results (-Fig. 1D).

\section{Turoctocog Alfa Pegol (Esperoct, Extended Half-Life Factor VIII)}

Turoctocog alfa pegol (Esperoct) is a recombinant FVIII molecule with site-specific (glyco)PEGylation within a truncated Bdomain. ${ }^{18}$ As shown in - Fig. 1E, turoctocog alfa pegol (Esper- oct) was underestimated at higher and lower tested concentrations by AFS and AFSL, respectively. In contrast, acceptable results over the whole concentration range were found for the FVIII:C CS as well as the PSL-based OSC assay.

\section{Extended Half-Life for Hemophilia B Treatment}

Measured FIX:C activities relative to EHL product input concentrations are described below and summarized in - Fig. 2 .

\section{Albutrepenonacog Alfa (Idelvion, Extended Half-Life Factor IX)}

Albutrepenonacog alfa (Idelvion) is a recombinant fusion protein that links a FIX molecule with albumin. ${ }^{19}$ With respect to the applied OSC assays, Idelvion was found to be underestimated by AFS, while AFSL showed threshold results. In 

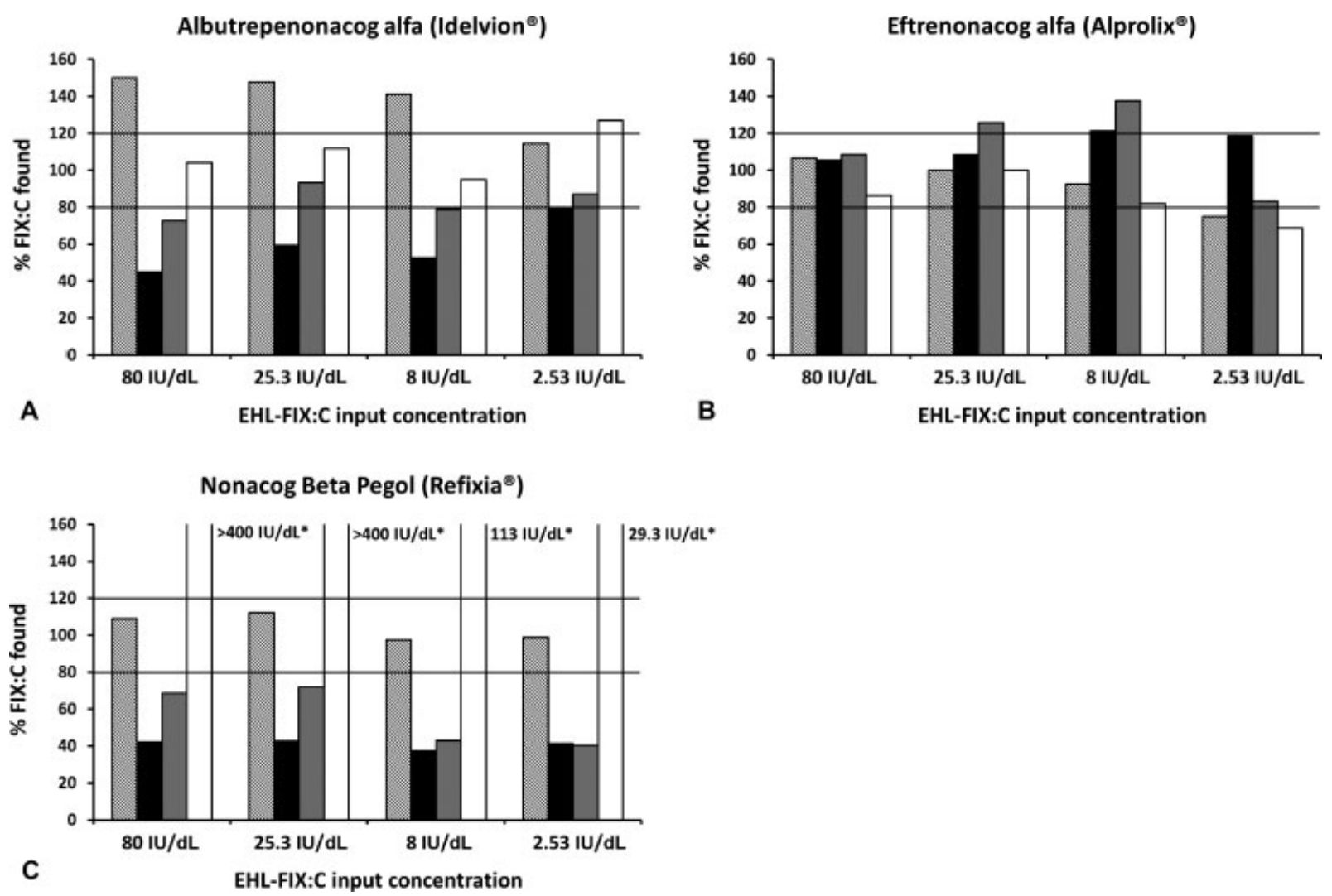

Fig. 2 Found FIX activities relative to EHL-FIX input concentrations. (A) Albutrepenonacog alfa (Idelvion). (B) Eftrenonacog alfa (Alprolix). (C) Nonacog beta pegol (Refixia). "Measured absolute values. Applied test systems (from left to right)-structured columns: FIX-CSA, black columns: FIX-OSC (AFS), gray columns: FIX-OSC (AFSL), white columns: FIX-OSC (PSL). The horizontal lines represent the arbitrarily defined range of acceptance $( \pm 20 \%)$.

contrast, PSL demonstrated acceptable results at the higher and intermediate concentrations, but overestimated Idelvion at the low input concentration. Regarding the FIX CS assay, the molecule was overestimated at higher and intermediate input concentrations ( - Fig. 2A). Therefore, the FIX:C OSC assay with PSL would be our recommendation.

Eftrenonacog Alfa (Alprolix, Extended Half-Life Factor IX) Eftrenonacog alfa (Alprolix) is a recombinant fusion protein that combines a FIX molecule with the Fc fragment of human $\operatorname{IgG1.}{ }^{20}$ As demonstrated in -Fig. 2B, the measurement of Alprolix revealed moderate, concentration-dependent effects for all test systems, whereas the FVIII-OSC using AFS showed the most accurate results.

Nonacog Beta Pegol (Refixia, Extended Half-Life Factor IX) Nonacog beta pegol (Refixia) is a recombinant, site-specific (glyco)PEGylated FIX molecule. ${ }^{21}$ Regarding the OSC assays, the experiments revealed (concentration-dependent) underestimation of the molecule by AFS as well as by AFSL, while the application of PSL demonstrated substantial overestimation of FIX activities. Indeed, only the FIX CS assay yielded acceptable results (-Fig. 2C).

Implementation of Extended Half-Life Product-Specific Analysis of Postinfusion Samples

As demonstrated earlier, the advent of FVIII- and FIX-EHL concentrates complicated the monitoring of corresponding factor-replacement therapies in both hemophilia A and B. Accordingly, based on the present results, each EHL product was allocated to an appropriate assay to prevent over- or underestimation of plasma factor activities (-Table 1). In order to allow for targeted laboratory requests, corresponding EHL product-specific analytes were defined and made available within the order entry system of our university hospital.

\section{Discussion}

In the present in vitro study, using a Siemens Atellica COAG 360 analyzer, we assessed the accuracy of different OSC and CS assays with respect to the determination of plasma EHLFVIII or -FIX activities. As expected, discrepancies of test results were observed that consequently necessitated EHLproduct-specific allocations of suitable assays. Meanwhile, the fact that the introduced modifications, in comparison to pFVIII applied for assay calibration, lead to altered activation and/or activity patterns of the EHL molecules within artificial in vitro test systems has been widely taken into account by manufacturers, coagulation laboratories, and relevant medical societies. This awareness is proven by the quantity of conducted field and also by smaller, local studies and the continuously growing number of corresponding original articles, reviews, and guidelines. ${ }^{11,12,18,22-25}$

The potential applicability of available OSC (aPTT reagents) or CS assays for the accurate determination of particular EHL 
Table 1 Summary of EHL products and allocated assays

\begin{tabular}{|l|l|l|}
\hline EHL product & Molecule characteristics & Allocated assay \\
\hline Efmoroctocog alfa (Elocta) & FVIII-Fc fusion protein & FVIII-OSC (AFS) \\
\hline Damoctocog alfa pegol (jivi) & FVIII w site-specific PEGylation & FVIII-OSC (AFSL) \\
\hline Lonoctocog alfa (Afstyla) & Single-chain FVIII molecule & FVIII-CSA \\
\hline Rurioctocog alfa pegol (Adynovi) & PEGylated FVIII (mainly B-domain) & FVIII-CSA \\
\hline Turoctocog alfa pegol (Esperoct) & FVIII w site-specific (glyco)PEGylation & FVIII-CSA \\
\hline Albutrepenonacog alfa (Idelvion) & FIX-albumin fusion protein & FIX-OSC (PSL) \\
\hline Eftrenonacog alfa (Alprolix) & FIX-Fc fusion protein & FIX-OSC (AFS) \\
\hline Nonacog beta pegol (Refixia) & FIX w site-specific (glyco)PEGylation & FIX-CSA \\
\hline
\end{tabular}

Abbreviations: EHL, extended half-life; OSC, one-stage clotting.

factor activity may be assessed by studying corresponding publications. In fact, the impact of several EHL products, especially on particular OSC assays, is to such an extent that resulting mismeasurements occur independent of the applied assay platform. This is especially true for Nonacog beta pegol (Refixia), for which it could be demonstrated that the use of silica-based aPTT reagents leads to premature activation of the pegylated FIX molecule before recalcification, resulting in artificially shortened clotting times, and hence, massive overestimation of plasma FIX:C activity. ${ }^{24}$ Furthermore, underestimation of lonoctocog alfa (Afstyla) by OSC assays by approximately $50 \%$ appears to be a general phenomenon, coming along in a moroctocog alfa (Refacto)-like fashion. ${ }^{8,9,25}$ For Afstyla, the activation profile by thrombin may be impaired which is overcome by chromogenic assays with their relative high concentration of purified thrombin compared to OSC assays. Indeed, due to corresponding specifications of the European Pharmacopoeia (Ph. Eur.), potency labeling of (EHL)-FVIII concentrates released in Europe is generally done using CS assays. ${ }^{26}$ Thus, one might expect that the application of a FVIII CS assay for determination of (EHL)-FVIII:C activity in postinfusion samples leads to accurate results. Interestingly, this is not generally true. For example, as described earlier and also demonstrated here, Efmoroctocog alfa (Elocta) is overestimated in postinfusion samples when measured using CS assays. ${ }^{27}$ While overestimations appear to be within clinically acceptable ranges, observed discrepancies probably stem from differences between World Health Organization (WHO) international standards for FVIII concentrate, used for potency labeling, and for FVIII (and vWF) in plasma, used for postinfusion measurements. ${ }^{27}$ Furthermore, it was recently shown that Damoctocog alfa pegol (Jivi) is underestimated by the Siemens CS assay, as also shown in the present study, but accurately assayed when using the BIOPHEN FVIII:C CS assay (Hyphen-Biomed). ${ }^{28}$ This finding also demonstrates that different compositions of FVIII CS assays (e.g., bovine vs. human factors, buffer formulations) may cause discrepant results during (EHL)-FVIII:C activity measurements.

In addition to the reagent-dependent effects discussed earlier, discrepant results may also be found when using the same reagent on different analyzers, with different calibrators and/or in different laboratories. ${ }^{29}$ Accordingly, there are also inconsistent publications on the performance of differ- ent reagents/assays with respect to plasma EHL factor measurements. ${ }^{23,30,31}$ Also the results of the present study may not be directly transferable to other laboratories using the same analyzers. Furthermore, the arbitrarily defined acceptance range of $+/-20 \%$ to determine over- or underestimation of test results may be chosen more broadly in other contexts. Thus, it appears reasonable that laboratories execute local studies to assess the performance of available assays and to initiate corresponding measures.

In the present study, EHL concentrates were reconstituted and spiked at different final concentrations into the corresponding factor-deficient plasma. While the presented findings are in accordance with previous reports, the followed strategy comes with potential limitations. On the one hand, immuno-adsorbed rather than congenital factor-deficient plasma was used for sample preparation. This may have had an impact on assay results (e.g., due to reduced vWF activity/antigen ratios). ${ }^{32}$ Furthermore, only one single batch of OSC or CS assay reagents was used during analysis, blocking the view on potential lot-specific differences. Thus, corresponding additional analysis may be performed to further substantiate the found results.

Nevertheless, based on the present findings, in addition to the established generic FVIII or FIX analytes, EHL-productspecific options were also defined and made available to clinicians to ensure the application of suitable assays and diagnostically conclusive results a priori. In daily clinical practice, however, targeted ordering for patients on EHL products is a complex task prone to errors, potentially leading to implausible results or, if unrecognized, even to clinical misinterpretations. The reasons for continued ordering of generic FVIII or FIX activities range from lack of knowledge that the patient is treated with an EHL product (or with which) over poor lines of communication to simple force of habit. Thus, in addition to correspondingly adopted/ extended ordering systems, continuous education and communication between treating physicians and the coagulation laboratory remain key elements with growing importance.

\section{Conflict of Interest}

J.M. reports nonfinancial support from Bayer Vital GmbH, CSL Behring, Novo Nordisk, Sobi, and Takeda, during the conduct of the study; personal fees from Siemens 
Healthineers, Novo Nordisk, and Bayer Vital GmbH, outside the submitted work.

\section{Acknowledgements}

The authors thank Daniela Krause and Simone Gasper for expert technical assistance.

\section{References}

1 Bolton-Maggs PH, Pasi KJ. Haemophilias A and B. Lancet 2003;361 (9371):1801-1809

2 Franchini M, Mannucci PM. The history of hemophilia. Semin Thromb Hemost 2014;40(05):571-576

3 Schramm W. The history of haemophilia - a short review. Thromb Res 2014;134(Suppl 1):S4-S9

4 Adcock DM, Strandberg K, Shima M, Marlar RA. Advantages, disadvantages and optimization of one-stage and chromogenic factor activity assays in haemophilia A and B. Int J Lab Hematol 2018;40(06):621-629

5 Oldenburg J, Pavlova A. Discrepancy between one-stage and chromogenic factor VIII activity assay results can lead to misdiagnosis of haemophilia A phenotype. Hamostaseologie 2010;30(04):207-211

6 Pavlova A, Delev D, Pezeshkpoor B, Müller J, Oldenburg J. Haemophilia A mutations in patients with non-severe phenotype associated with a discrepancy between one-stage and chromogenic factor VIII activity assays. Thromb Haemost 2014;111(05):851-861

7 Pezeshkpoor B, Gazorpak M, Berkemeier AC, et al. In silico and in vitro evaluation of the impact of mutations in non-severe haemophilia A patients on assay discrepancies. Ann Hematol 2019;98 (08):1855-1865

8 Mikaelsson M, Oswaldsson U, Sandberg H. Influence of phospholipids on the assessment of factor VIII activity. Haemophilia 1998; 4(04):646-650

9 Mikaelsson M, Oswaldsson U, Jankowski MA. Measurement of factor VIII activity of B-domain deleted recombinant factor VIII. Semin Hematol 2001;38(02, Suppl 4):13-23

10 Wilmot HV, Hogwood J, Gray E. Recombinant factor IX: discrepancies between one-stage clotting and chromogenic assays. Haemophilia 2014;20(06):891-897

11 Chowdary P. Extended half-life recombinant products in haemophilia clinical practice - expectations, opportunities and challenges. Thromb Res 2019 (epub ahead of print). Doi: 10.1016/j. thromres.2019.12.012

12 Peyvandi F, Kenet G, Pekrul I, Pruthi RK, Ramge P, Spannagl M. Laboratory testing in hemophilia: impact of factor and non-factor replacement therapy on coagulation assays. J Thromb Haemost 2020;18(06):1242-1255

13 Hörber S, Lehmann R, Peter A. Evaluation of the Atellica COAG 360 coagulation analyzer in a central laboratory of a maximum care hospital. Int J Lab Hematol 2020;42(01):28-36

14 Frampton JE. Efmoroctocog alfa: a review in haemophilia A. Drugs 2016;76(13):1281-1291

15 Paik J, Deeks ED. Damoctocog alfa pegol: a review in haemophilia A. Drugs 2019;79(10):1147-1156

16 Al-Salama ZT, Scott LJ. Lonoctocog alfa: a review in haemophilia A. Drugs 2017;77(15):1677-1686

17 Konkle BA, Stasyshyn O, Chowdary P, et al. Pegylated, fulllength, recombinant factor VIII for prophylactic and on-demand treatment of severe hemophilia A. Blood 2015;126(09):10781085

18 Ezban M, Hansen M, Kjalke M. An overview of turoctocog alfa pegol (N8-GP; ESPEROCT ${ }^{\circledR}$ ) assay performance: Implications for postadministration monitoring. Haemophilia 2020;26(01): 156-163

19 Lyseng-Williamson KA. Coagulation factor IX (Recombinant), albumin fusion protein (albutrepenonacog alfa; Idelvion ${ }^{\circledR}$ ): a review of its use in haemophilia B. Drugs 2017;77(01):97-106

20 Hoy SM. Eftrenonacog alfa: a review in haemophilia B. Drugs 2017;77(11):1235-1246

21 Syed YY. Nonacog beta pegol: a review in haemophilia B. Drugs 2017;77(18):2003-2012

22 Young GA, Perry DJInternational Prophylaxis Study Group (IPSG). Laboratory assay measurement of modified clotting factor concentrates: a review of the literature and recommendations for practice. J Thromb Haemost 2019;17(04):567-573

23 Gray E, Kitchen S, Bowyer A, et al. Laboratory measurement of factor replacement therapies in the treatment of congenital haemophilia: a United Kingdom Haemophilia Centre Doctors' Organisation guideline. Haemophilia 2020;26(01): 6-16

24 Rosén P, Rosén S, Ezban M, Persson E. Overestimation of NglycoPEGylated factor IX activity in a one-stage factor IX clotting assay owing to silica-mediated premature conversion to activated factor IX. J Thromb Haemost 2016;14(07): $1420-1427$

25 St Ledger K, Feussner A, Kalina U, et al. International comparative field study evaluating the assay performance of AFSTYLA in plasma samples at clinical hemostasis laboratories. J Thromb Haemost 2018;16(03):555-564

26 Dodt J, Hubbard AR, Wicks SJ, et al. Potency determination of factor VIII and factor IX for new product labelling and postinfusion testing: challenges for caregivers and regulators. Haemophilia 2015;21(04):543-549

27 Sommer JM, Moore N, McGuffie-Valentine B, et al. Comparative field study evaluating the activity of recombinant factor VIII FC fusion protein in plasma samples at clinical haemostasis laboratories. Haemophilia 2014;20(02):294-300

28 Meijer P, Marlar RA, Teare JM, Adcock D. Inter-laboratory evaluation of the recovery of Bay 94-9027 [Jivi®] with one-stage clotting and chromogenic assays. Blood 2019;134(Suppl 1):1124

29 Hillarp A, Bowyer A, Ezban M, Persson P, Kitchen S. Measuring FVIII activity of glycopegylated recombinant factor VIII, N8-GP, with commercially available one-stage clotting and chromogenic assay kits: a two-centre study. Haemophilia 2017;23(03): 458-465

30 Turecek PL, Romeder-Finger S, Apostol C, et al. A world-wide survey and field study in clinical haemostasis laboratories to evaluate FVIII:C activity assay variability of ADYNOVATE and OBIZUR in comparison with ADVATE. Haemophilia 2016;22 (06):957-965

31 Bulla O, Poncet A, Alberio L, et al. Impact of a product-specific reference standard for the measurement of a PEGylated rFVIII activity: the Swiss Multicentre Field Study. Haemophilia 2017;23 (04):e335-e339

32 Butenas S, Parhami-Seren B, Undas A, Fass DN, Mann KG. The "normal" factor VIII concentration in plasma. Thromb Res 2010; 126(02):119-123 